\title{
Effect of BG-II Cotton Hybrids and Non Bt Cotton on Weight of Different Instars of Spodoptera litura (FAB.)
}

\author{
T. Ramanjali ${ }^{1 *}$, T.V.K. Singh ${ }^{2 *}$ and C. Narendra Reddy ${ }^{1}$ \\ ${ }^{1}$ Department of Entomology, College of agriculture, Rajendranagar, \\ Hyderabad-500030, Telangana, India \\ ${ }^{2}$ Department of Agriculture, Professor Jayashankar Telangana State Agricultural University, \\ Rajendranagar, Hyderabad-500030, Telangana, India \\ *Corresponding author
}

\begin{tabular}{|c|c|}
\hline \multicolumn{2}{|r|}{ A B S T R A C T } \\
\hline & \multirow{5}{*}{$\begin{array}{l}\text { Laboratory assays showed that dual toxin Bacillus thuringiensis (Bt) cotton } \\
\text { cultivars expressing both Cry } 1 \text { Ac and Cry } 2 \text { Ab endotoxins (BG-II) were } \\
\text { more toxic to Spodoptera litura (Fab.) larvae compared to non Bt cotton. } \\
\text { The weight of first, second, third and fourth instar larvae were recorded, } \\
\text { which were fed with leaves, squares and bolls of eleven dual toxin Bt } \\
\text { cotton cultivars at } 60,75,90 \text { and } 125 \text { days after sowing of the crop. The } \\
\text { final weight of each instar was reduced at seven days after release when } \\
\text { compared to non Bt cotton cultivars. Reduction in the weight was more in } \\
\text { case of first, second and third instars than the fourth instar larvae of } S \text {. } \\
\text { litura. Hence the BG-II cotton was more toxic to first, second, third instar } \\
\text { larvae than fourth instar. }\end{array}$} \\
\hline & \\
\hline & \\
\hline $\mathbf{A r}$ & \\
\hline $\begin{array}{l}\text { Accepted: } \\
21 \text { Septem } \\
\text { Available } \\
10 \text { October }\end{array}$ & \\
\hline
\end{tabular}

\section{Introduction}

Transgenic cotton, Gossypium hirsutum (L.), expressing insecticidal $\delta$-endotoxin protein of Bacillus thuringiensis Berliner, hereafter referred to as Bt cotton ${ }^{1}$. The introduction of $\mathrm{Bt}$ transgenic cotton hybrids in the year 200203 in all the cotton growing tracts of Andhra Pradesh revolutionized boll worm management of cotton resulting in a total shift in pesticide use pattern in the crop $^{2}$. The single (Cry 1 Ac) gene Bt transgenic hybrids are highly resistant to boll worms, especially American boll worm, Helicoverpa armigera affording very good protection from this pest.
However, the incidence of tobacco caterpillar, $S$. litura has been noticed on Bt cotton hybrids during vegetative phage to levels necessitating use of pesticide sprays to manage the pest. So, the BG-I cotton is slowly replaced by BG-II expressing dual genes (Cry 1Ac+Cry2Ab). Bollgard-II cotton expressing both Cry $1 \mathrm{Ac}$ and Cry $2 \mathrm{Ab}$ proteins have provided increased efficacy against the budworm-bollworm complex and enhanced spectrum of activity against beet armyworm, Spodoptera exigua (Hub.) fall armyworm, Spodoptera frugiperda (Smith) 
and common cutworm or tobacco caterpillar Spodoptera litura (Fab.) which have been predicted to be major pests in emerging scenario $^{3,4,5}$. The present study aims to understand the effect of double gene $\mathrm{Bt}$ transgenic cotton hybrids and non Bt cotton on the weight of different larval instars of $S$. litura through laboratory studies.

\section{Materials and Methods}

Laboratory experiments were carried out during 2013-14 to study the effect of eleven BG-II cotton hybrids viz., Ajeeth-155, Ankur3034, Chetak, ATM, Bhakti, Brahma, Rasi665, Rasi-668, Denim, Sudarshan, Yuva and non Bt cotton plant parts (leaves, squares and bolls) as control against first, second, third and fourth instar larvae of S. litura at 60, 75, 90 and 125 DAS.

Eleven BG-II cotton hybrids and non $\mathrm{Bt}$ cotton plots were maintained at College Farm, College of Agriculture, Rajendranagar, Hyderabad during Kharif-2013. Field plots were maintained by standard agronomic practices, including herbicide and fertilizer applications.
Rearing of S. litura culture was carried out at BT lab, Department of Entomology, College of Agriculture, Rajendranagar, Hyderabad. Initially the culture was collected from the field on castor plants. After S. litura larvae were reared on castor leaves in BT lab. Immediately after first generation the experiments was conducted with the first, second, third and fourth instar larvae.

Leaves, squares and bolls were plucked with $0.5 \mathrm{~cm}$ of petiole remaining to prevent desiccation from the crop of 60, 75, 90 and 125 days old dual toxin Bt cotton hybrids and non $\mathrm{Bt}$ cotton. Later these were cleaned with distilled water and wiped off with blotting paper to remove excess moisture and air dried. The cleaned leaves, squares and bolls were placed after suitably trimming (to fit into the cups) individually into rearing cups. Within 1-2 h of harvesting, a single leaf/ 3-4 squares/ one boll were placed into individual rearing cups and larvae were placed into each cup. S. litura larval instars cultured on natural diet were used, three replications of 15 larvae for each treatment kept in rearing cups at ambient temperature in laboratory.

\section{Treatments}

\begin{tabular}{|c|c|c|c|c|}
\hline Instars/treatmnets & First Instar & Second Instar & Third Instar & Fourth Instar \\
\hline \multirow{3}{*}{$\begin{array}{l}\text { Treatments (Eleven BG- } \\
\text { II hybrids) }\end{array}$} & $\begin{array}{l}\text { Leaves }(60,75,90 \\
\text { and } 125 \text { DAS })\end{array}$ & $\begin{array}{l}\text { Leaves }(60,75,90 \\
\text { and } 125 \text { DAS) }\end{array}$ & $\begin{array}{c}\text { Leaves }(60,75, \\
90 \text { and } 125 \\
\text { DAS })\end{array}$ & $\begin{array}{c}\text { Leaves }(60,75, \\
90 \text { and } 125 \\
\text { DAS })\end{array}$ \\
\hline & \multirow{2}{*}{$\begin{array}{l}\text { Squares }(60,75,90 \\
\text { and } 125 \text { DAS })\end{array}$} & $\begin{array}{l}\text { Squares }(60,75,90 \\
\text { and } 125 \text { DAS })\end{array}$ & $\begin{array}{c}\text { Squares }(60, \\
75,90 \text { and } 125 \\
\text { DAS })\end{array}$ & $\begin{array}{c}\text { Squares }(60, \\
75,90 \text { and } 125 \\
\text { DAS })\end{array}$ \\
\hline & & $\begin{array}{c}\text { Bolls (90 and } 125 \\
\text { DAS) }\end{array}$ & $\begin{array}{l}\text { Bolls (90 and } \\
125 \text { DAS) }\end{array}$ & $\begin{array}{l}\text { Bolls (90 and } \\
125 \text { DAS) }\end{array}$ \\
\hline \multirow{3}{*}{ Control (Non Bt cotton) } & $\begin{array}{l}\text { Leaves }(60,75,90 \\
\text { and } 125 \text { DAS) }\end{array}$ & $\begin{array}{l}\text { Leaves }(60,75,90 \\
\text { and } 125 \text { DAS) }\end{array}$ & $\begin{array}{c}\text { Leaves }(60,75, \\
90 \text { and } 125 \\
\text { DAS })\end{array}$ & $\begin{array}{c}\text { Leaves }(60,75, \\
90 \text { and } 125 \\
\text { DAS })\end{array}$ \\
\hline & \multirow{2}{*}{$\begin{array}{l}\text { Squares }(60,75,90 \\
\text { and } 125 \text { DAS })\end{array}$} & $\begin{array}{l}\text { Squares }(60,75,90 \\
\text { and } 125 \text { DAS })\end{array}$ & $\begin{array}{c}\text { Squares }(60, \\
75,90 \text { and } 125 \\
\text { DAS })\end{array}$ & $\begin{array}{c}\text { Squares }(60, \\
75,90 \text { and } 125 \\
\text { DAS })\end{array}$ \\
\hline & & $\begin{array}{c}\text { Bolls (90 and } 125 \\
\text { DAS) }\end{array}$ & $\begin{array}{l}\text { Bolls (90 and } \\
125 \text { DAS) }\end{array}$ & $\begin{array}{l}\text { Bolls (90 and } \\
125 \text { DAS) }\end{array}$ \\
\hline
\end{tabular}


Fresh leaves, squares and bolls collected as before, were provided to larvae up to seven days after release. Seven days after release the survived larval weight was recorded.

The data recorded on weight of $S$. litura larvae on Bollgard-II cotton were subjected to completely randomized design (CRD) ${ }^{6}$. The CRD analysis was done by using the statistical programme opstat.

\section{Results and Discussion}

First instar larvae of $S$. litura were more susceptible to leaves and squares of dual toxin hybrids than non Bt cotton. The weight of the first instar larvae was not recorded at seven days after release due to the 100 per cent mortality of first instar larvae on leaves of BG-II cotton.

The weight of second, third and fourth instar larvae of $S$. litura fed with leaves of eleven BG-II cotton hybrids was lowest when compared to non-Bt leaves (Table 1).

Minimum weight of second, third and fourth instar larvae fed with leaves of BG-II cotton hybrids recorded as $0.021,0.055$ and $0.173 \mathrm{~g}$ per larvae, respectively at seven days after release.

Similar results were also obtained by $\mathrm{Naik}^{7}$ where in $0.016,0.035$ and $0.059 \mathrm{~g}$ per larval weight was observed in S. litura larvae fed with BG-II cotton hybrid leaves at $72 \mathrm{~h}$ after release.

The lowest weight of first, second, third and fourth instar larvae of $S$. litura fed with leaves were recorded on Rasi-665 BG-II hybrid. These observations were similar with the results of Soujanya ${ }^{8}$ the weight of the larvae fed with dual toxin Bt cultivars was significantly reduced compared to the larvae fed with $\mathrm{Bt}$ and non-Bt cultivars and the reduction in the larval weight progressively increased from 24 to $72 \mathrm{~h}$ of feeding up to fifth instar and there was no significant difference in larval weights that were fed with Bt and non-Bt hybrids.

\section{Squares}

Minimum weight of first, second, third and fourth instar larvae fed with squares of BG-II cotton hybrids was recorded as 0.009, 0.020, 0.055 and $0.175 \mathrm{~g}$ per larvae at seven days after release in between 60 and 125 DAS (Tables 2 and 3).

Similar results were also obtained by $\mathrm{Naik}^{7}$, where the weight of second, third and fourth instar larvae was $0.020,0.039$ and $0.066 \mathrm{~g}$ per larvae when fed with BGII cotton hybrids squares at $72 \mathrm{~h}$ after release.

Among eleven BG-II cotton hybrids Rasi-665 recorded lowest weight on squares at 60,75 , 90 and 125 DAS. On non-Bt the weight was more when compared to eleven BG-II cotton hybrids. The weight was less on squares, compared to leaves. These findings are in line with the results of Naik ${ }^{7}$.

\section{Bolls}

Minimum weight of second, third and fourth instar larvae fed with bolls of BG-II cotton hybrid was recorded as 0.017, 0.053 and $0.191 \mathrm{~g}$ per larvae at seven days after release in between 60 and 125 DAS (Table 4).

Among all eleven BG-II cotton hybrids minimum weight of second, third and fourth instars at 90 and 125 DAS was recorded on bolls of Rasi-665.

On leaves, squares and bolls the weight of first, second, third and fourth instar larvae on BG-II cotton hybrids was significantly different from non-Bt cotton. 
Table.1 Effect of test hybrid leaves on larval weight of S. litura.

\begin{tabular}{|c|c|c|c|c|c|c|c|c|c|c|c|c|}
\hline \multirow{2}{*}{ Treatments } & \multicolumn{4}{|c|}{ Weight of Second Instar larvae (g) } & \multicolumn{4}{|c|}{ Weight of third Instar larvae (g) } & \multicolumn{4}{|c|}{ Weight of fourth Instar larvae (g) } \\
\hline & 60 DAS & 75 DAS & 90 DAS & 125 DAS & 60 DAS & 75 DAS & 90 DAS & 125 DAS & 60 DAS & 75 DAS & 90 DAS & 125 DAS \\
\hline Ankur-3034 & $\begin{array}{c}0.037 \\
(1.02)^{\mathrm{a}}\end{array}$ & $\begin{array}{c}0.045 \\
(1.02)^{\mathrm{a}}\end{array}$ & $\begin{array}{c}0.050 \\
(1.03)^{\mathrm{a}}\end{array}$ & $\begin{array}{c}0.034 \\
(1.02)^{\mathrm{a}}\end{array}$ & $\begin{array}{c}0.082 \\
(1.04)^{\mathrm{a}}\end{array}$ & $\begin{array}{c}0.070 \\
(1.04)^{\mathrm{a}}\end{array}$ & $\begin{array}{c}0.120 \\
(1.06)^{\mathrm{a}}\end{array}$ & $\begin{array}{c}0.101 \\
(1.05)^{\mathrm{a}}\end{array}$ & $\begin{array}{c}0.192 \\
(1.09)^{\mathrm{a}}\end{array}$ & $\begin{array}{c}0.218 \\
(1.10)^{\mathrm{a}}\end{array}$ & $\begin{array}{c}0.248 \\
(1.12)^{\mathrm{a}}\end{array}$ & $\begin{array}{c}0.224 \\
(1.11)^{\mathrm{a}}\end{array}$ \\
\hline Ajeet-155 & $\begin{array}{c}0.046 \\
(1.02)^{\mathrm{a}} \\
\end{array}$ & $\begin{array}{c}0.042 \\
(1.02)^{\mathrm{a}} \\
\end{array}$ & $\begin{array}{c}0.032 \\
(1.02)^{\mathrm{a}} \\
\end{array}$ & $\begin{array}{c}0.036 \\
(1.02)^{\mathrm{a}}\end{array}$ & $\begin{array}{c}0.109 \\
(1.05)^{\mathrm{a}} \\
\end{array}$ & $\begin{array}{c}0.086 \\
(1.04)^{\mathrm{a}} \\
\end{array}$ & $\begin{array}{c}0.089 \\
(1.04)^{\mathrm{a}} \\
\end{array}$ & $\begin{array}{c}0.063 \\
(1.03)^{\mathrm{a}} \\
\end{array}$ & $\begin{array}{c}0.265 \\
(1.13)^{\mathrm{a}} \\
\end{array}$ & $\begin{array}{c}0.230 \\
(1.11)^{\mathrm{a}} \\
\end{array}$ & $\begin{array}{c}0.251 \\
(1.12)^{\mathrm{a}} \\
\end{array}$ & $\begin{array}{c}0.237 \\
(1.11)^{\mathrm{a}} \\
\end{array}$ \\
\hline Chetak & $\begin{array}{c}0.046 \\
(1.02)^{\mathrm{a}}\end{array}$ & $\begin{array}{c}0.034 \\
(1.02)^{\mathrm{a}}\end{array}$ & $\begin{array}{c}0.038 \\
(1.02)^{\mathrm{a}}\end{array}$ & $\begin{array}{c}0.032 \\
(1.02)^{\mathrm{a}}\end{array}$ & $\begin{array}{c}0.097 \\
(1.05)^{\mathrm{a}}\end{array}$ & $\begin{array}{c}0.086 \\
(1.04)^{\mathrm{a}} \\
\end{array}$ & $\begin{array}{c}0.087 \\
(1.04)^{\mathrm{a}}\end{array}$ & $\begin{array}{c}0.069 \\
(1.03)^{\mathrm{a}}\end{array}$ & $\begin{array}{c}0.281 \\
(1.13)^{\mathrm{a}}\end{array}$ & $\begin{array}{c}0.225 \\
(1.11)^{\mathrm{a}}\end{array}$ & $\begin{array}{c}0.248 \\
(1.12)^{\mathrm{a}} \\
\end{array}$ & $\begin{array}{c}0.233 \\
(1.11)^{\mathrm{a}} \\
\end{array}$ \\
\hline ATM & $\begin{array}{c}0.031 \\
(1.02)^{\mathrm{a}}\end{array}$ & $\begin{array}{c}0.038 \\
(1.02)^{\mathrm{a}}\end{array}$ & $\begin{array}{c}0.042 \\
(1.02)^{\mathrm{a}}\end{array}$ & $\begin{array}{c}0.036 \\
(1.02)^{\mathrm{a}}\end{array}$ & $\begin{array}{c}0.091 \\
(1.05)^{\mathrm{a}}\end{array}$ & $\begin{array}{c}0.069 \\
(1.03)^{\mathrm{a}}\end{array}$ & $\begin{array}{c}0.074 \\
(1.04)^{\mathrm{a}}\end{array}$ & $\begin{array}{c}0.062 \\
(1.03)^{\mathrm{a}}\end{array}$ & $\begin{array}{c}0.210 \\
(1.10)^{\mathrm{a}}\end{array}$ & $\begin{array}{c}0.201 \\
(1.10)^{\mathrm{a}}\end{array}$ & $\begin{array}{c}0.235 \\
(1.11)^{\mathrm{a}}\end{array}$ & $\begin{array}{c}0.224 \\
(1.11)^{\mathrm{a}}\end{array}$ \\
\hline Bhakti & $\begin{array}{c}0.023 \\
(1.01)^{\mathrm{a}} \\
\end{array}$ & $\begin{array}{c}0.038 \\
(1.02)^{\mathrm{a}} \\
\end{array}$ & $\begin{array}{c}0.037 \\
(1.02)^{\mathrm{a}} \\
\end{array}$ & $\begin{array}{c}0.025 \\
(1.01)^{\mathrm{a}}\end{array}$ & $\begin{array}{c}0.084 \\
(1.04)^{\mathrm{a}} \\
\end{array}$ & $\begin{array}{c}0.086 \\
(1.04)^{\mathrm{a}} \\
\end{array}$ & $\begin{array}{c}0.072 \\
(1.04)^{\mathrm{a}} \\
\end{array}$ & $\begin{array}{c}0.072 \\
(1.04)^{\mathrm{a}}\end{array}$ & $\begin{array}{c}0.254 \\
(1.12)^{\mathrm{a}} \\
\end{array}$ & $\begin{array}{c}0.221 \\
(1.11)^{\mathrm{a}} \\
\end{array}$ & $\begin{array}{c}0.209 \\
(1.10)^{\mathrm{a}} \\
\end{array}$ & $\begin{array}{c}0.231 \\
(1.10)^{\mathrm{a}} \\
\end{array}$ \\
\hline Brahma & $\begin{array}{c}0.028 \\
(1.01)^{\mathrm{a}}\end{array}$ & $\begin{array}{c}0.039 \\
(1.02)^{\mathrm{a}}\end{array}$ & $\begin{array}{c}0.040 \\
(1.020)^{\mathrm{a}}\end{array}$ & $\begin{array}{c}0.027 \\
(1.01)^{\mathrm{a}}\end{array}$ & $\begin{array}{c}0.093 \\
(1.05)^{\mathrm{a}}\end{array}$ & $\begin{array}{c}0.078 \\
(1.04)^{\mathrm{a}}\end{array}$ & $\begin{array}{c}0.129 \\
(1.06)^{\mathrm{a}}\end{array}$ & $\begin{array}{c}0.061 \\
(1.03)^{\mathrm{a}}\end{array}$ & $\begin{array}{c}0.210 \\
(1.10)^{\mathrm{a}}\end{array}$ & $\begin{array}{c}0.195 \\
(1.09)^{\mathrm{a}}\end{array}$ & $\begin{array}{c}0.215 \\
(1.10)^{\mathrm{a}}\end{array}$ & $\begin{array}{c}0.212 \\
(1.10)^{\mathrm{a}}\end{array}$ \\
\hline Denim & $\begin{array}{c}0.028 \\
(1.01)^{\mathrm{a}}\end{array}$ & $\begin{array}{c}0.022 \\
(1.01)^{\mathrm{a}}\end{array}$ & $\begin{array}{c}0.035 \\
(1.02)^{\mathrm{a}}\end{array}$ & $\begin{array}{c}0.032 \\
(1.02)^{\mathrm{a}}\end{array}$ & $\begin{array}{c}0.087 \\
(1.04)^{\mathrm{a}}\end{array}$ & $\begin{array}{c}0.072 \\
(1.04)^{\mathrm{a}}\end{array}$ & $\begin{array}{c}0.112 \\
(1.05)^{\mathrm{a}}\end{array}$ & $\begin{array}{c}0.058 \\
(1.03)^{\mathrm{a}}\end{array}$ & $\begin{array}{c}0.284 \\
(1.13)^{\mathrm{a}}\end{array}$ & $\begin{array}{c}0.187 \\
(1.09)^{\mathrm{a}}\end{array}$ & $\begin{array}{c}0.223 \\
(1.11)^{\mathrm{a}}\end{array}$ & $\begin{array}{c}0.217 \\
(1.10)^{\mathrm{a}}\end{array}$ \\
\hline Rasi-665 & $\begin{array}{c}0.021 \\
(1.01)^{\mathrm{a}} \\
\end{array}$ & $\begin{array}{c}0.021 \\
(1.01)^{\mathrm{a}} \\
\end{array}$ & $\begin{array}{c}0.025 \\
(1.01)^{\mathrm{a}} \\
\end{array}$ & $\begin{array}{c}0.025 \\
(1.01)^{\mathrm{a}}\end{array}$ & $\begin{array}{c}0.093 \\
(1.05)^{\mathrm{a}} \\
\end{array}$ & $\begin{array}{c}0.069 \\
(1.03)^{\mathrm{a}} \\
\end{array}$ & $\begin{array}{c}0.071 \\
(1.04)^{\mathrm{a}} \\
\end{array}$ & $\begin{array}{c}0.055 \\
(1.03)^{\mathrm{a}}\end{array}$ & $\begin{array}{c}0.191 \\
(1.09)^{\mathrm{a}} \\
\end{array}$ & $\begin{array}{c}0.175 \\
(1.08)^{\mathrm{a}} \\
\end{array}$ & $\begin{array}{c}0.173 \\
(1.08)^{\mathrm{a}} \\
\end{array}$ & $\begin{array}{c}0.175 \\
(1.10)^{\mathrm{a}} \\
\end{array}$ \\
\hline Rasi-668 & $\begin{array}{c}0.026 \\
(1.01)^{\mathrm{a}}\end{array}$ & $\begin{array}{c}0.027 \\
(1.01)^{\mathrm{a}}\end{array}$ & $\begin{array}{c}0.028 \\
(1.01)^{\mathrm{a}}\end{array}$ & $\begin{array}{c}0.027 \\
(1.01)^{\mathrm{a}}\end{array}$ & $\begin{array}{c}0.071 \\
(1.04)^{\mathrm{a}}\end{array}$ & $\begin{array}{c}0.082 \\
(1.04)^{\mathrm{a}}\end{array}$ & $\begin{array}{c}0.084 \\
(1.04)^{\mathrm{a}}\end{array}$ & $\begin{array}{c}0.061 \\
(1.03)^{\mathrm{a}}\end{array}$ & $\begin{array}{c}0.198 \\
(1.10)^{\mathrm{a}}\end{array}$ & $\begin{array}{c}0.198 \\
(1.10)^{\mathrm{a}}\end{array}$ & $\begin{array}{c}0.201 \\
(1.10)^{\mathrm{a}}\end{array}$ & $\begin{array}{c}0.217 \\
(1.10)^{\mathrm{a}}\end{array}$ \\
\hline Sudarshan & $\begin{array}{c}0.025 \\
(1.01)^{\mathrm{a}}\end{array}$ & $\begin{array}{c}0.036 \\
(1.02)^{\mathrm{a}}\end{array}$ & $\begin{array}{c}0.034 \\
(1.02)^{\mathrm{a}}\end{array}$ & $\begin{array}{c}0.040 \\
(1.02)^{\mathrm{a}}\end{array}$ & $\begin{array}{c}0.101 \\
(1.05)^{\mathrm{a}}\end{array}$ & $\begin{array}{c}0.078 \\
(1.04)^{\mathrm{a}}\end{array}$ & $\begin{array}{c}0.083 \\
(1.04)^{\mathrm{a}}\end{array}$ & $\begin{array}{c}0.083 \\
(1.04)^{\mathrm{a}}\end{array}$ & $\begin{array}{c}0.252 \\
(1.12)^{\mathrm{a}}\end{array}$ & $\begin{array}{c}0.189 \\
(1.10)^{\mathrm{a}}\end{array}$ & $\begin{array}{c}0.208 \\
(1.10)^{\mathrm{a}}\end{array}$ & $\begin{array}{c}0.177 \\
(1.09)^{\mathrm{a}}\end{array}$ \\
\hline Yuva & $\begin{array}{c}0.026 \\
(1.01)^{\mathrm{a}}\end{array}$ & $\begin{array}{c}0.043 \\
(1.02)^{\mathrm{a}}\end{array}$ & $\begin{array}{c}0.033 \\
(1.02)^{\mathrm{a}}\end{array}$ & $\begin{array}{c}0.053 \\
(1.03)^{\mathrm{a}}\end{array}$ & $\begin{array}{c}0.087 \\
(1.04)^{\mathrm{a}}\end{array}$ & $\begin{array}{c}0.081 \\
(1.04)^{\mathrm{a}}\end{array}$ & $\begin{array}{c}0.089 \\
(1.04)^{\mathrm{a}}\end{array}$ & $\begin{array}{c}0.091 \\
(1.05)^{\mathrm{a}}\end{array}$ & $\begin{array}{c}0.226 \\
(1.11)^{\mathrm{a}}\end{array}$ & $\begin{array}{c}0.242 \\
(1.11)^{\mathrm{a}}\end{array}$ & $\begin{array}{c}0.185 \\
(1.09)^{\mathrm{a}}\end{array}$ & $\begin{array}{c}0.233 \\
(1.11)^{\mathrm{a}}\end{array}$ \\
\hline Control & $\begin{array}{c}0.032 \\
(1.02)^{\mathrm{a}}\end{array}$ & $\begin{array}{c}0.048 \\
(1.02)^{\mathrm{a}}\end{array}$ & $\begin{array}{c}0.033 \\
(1.02)^{\mathrm{a}}\end{array}$ & $\begin{array}{c}0.038 \\
(1.02)^{\mathrm{a}}\end{array}$ & $\begin{array}{c}0.09 \\
(1.04)^{\mathrm{a}} \\
\end{array}$ & $\begin{array}{c}0.087 \\
(1.04)^{\mathrm{a}}\end{array}$ & $\begin{array}{c}0.096 \\
(1.05)^{\mathrm{a}}\end{array}$ & $\begin{array}{c}0.084 \\
(1.04)^{\mathrm{a}}\end{array}$ & $\begin{array}{c}0.231 \\
(1.11)^{\mathrm{a}}\end{array}$ & $\begin{array}{c}0.287 \\
(1.13)^{\mathrm{a}} \\
\end{array}$ & $\begin{array}{c}0.239 \\
(1.11)^{\mathrm{a}} \\
\end{array}$ & $\begin{array}{c}0.256 \\
(1.12)^{\mathrm{a}}\end{array}$ \\
\hline CD & 0.004 & 0.005 & 0.004 & 0.003 & 0.004 & 0.005 & 0.009 & 0.003 & 0.026 & 0.01 & 0.005 & 0.008 \\
\hline SE (m) & 0.002 & 0.002 & 0.001 & 0.001 & 0.001 & 0.002 & 0.003 & 0.001 & 0.01 & 0.004 & 0.002 & 0.003 \\
\hline
\end{tabular}

Figures in parentheses are square root transformed values; numbers followed by same superscript are not statistically different.

*First instar recorded 100 per cent mortality. 
Table.2 Effect of test hybrid squares on weight of first and second instar larvae of S. litura.

\begin{tabular}{|c|c|c|c|c|c|c|c|c|}
\hline \multirow{2}{*}{ Treatments } & \multicolumn{4}{|c|}{ Weight of first Instar larvae (g) } & \multicolumn{4}{|c|}{ Weight of second Instar larvae (g) } \\
\hline & 60 DAS & 75 DAS & 90 DAS & 125 DAS & 60 DAS & 75 DAS & 90 DAS & $125 \mathrm{DAS}$ \\
\hline Ankur-3034 & $\begin{array}{c}0.010 \\
(1.01)^{\mathrm{a}}\end{array}$ & $\begin{array}{c}0.010 \\
(1.01)^{\mathrm{a}}\end{array}$ & $\begin{array}{c}0.011 \\
(1.01)^{\mathrm{a}}\end{array}$ & $\begin{array}{c}0.009 \\
(1.01)^{\mathrm{a}}\end{array}$ & $\begin{array}{c}0.028 \\
(1.01)^{\mathrm{a}}\end{array}$ & $\begin{array}{c}0.024 \\
(1.01)^{\mathrm{a}}\end{array}$ & $\begin{array}{c}0.038 \\
(1.02)^{\mathrm{a}}\end{array}$ & $\begin{array}{c}0.033 \\
(1.02)^{\mathrm{a}}\end{array}$ \\
\hline Ajeet-155 & $\begin{array}{c}0.015 \\
(1.01)^{\mathrm{a}}\end{array}$ & $\begin{array}{c}0.013 \\
(1.01)^{\mathrm{a}}\end{array}$ & $\begin{array}{c}0.012 \\
(1.01)^{\mathrm{a}}\end{array}$ & $\begin{array}{c}0.012 \\
(1.01)^{\mathrm{a}}\end{array}$ & $\begin{array}{c}0.052 \\
(1.03)^{\mathrm{a}}\end{array}$ & $\begin{array}{c}0.039 \\
(1.02)^{\mathrm{a}} \\
\end{array}$ & $\begin{array}{c}0.041 \\
(1.02)^{\mathrm{a}}\end{array}$ & $\begin{array}{c}0.036 \\
(1.02)^{\mathrm{a}}\end{array}$ \\
\hline Chetak & $\begin{array}{c}0.011 \\
(1.01)^{\mathrm{a}}\end{array}$ & $\begin{array}{c}0.009 \\
(1.00)^{\mathrm{a}}\end{array}$ & $\begin{array}{c}0.010 \\
(1.01)^{\mathrm{a}}\end{array}$ & $\begin{array}{c}0.011 \\
(1.01)^{\mathrm{a}}\end{array}$ & $\begin{array}{c}0.038 \\
(1.02)^{\mathrm{a}}\end{array}$ & $\begin{array}{c}0.033 \\
(1.02)^{\mathrm{a}}\end{array}$ & $\begin{array}{c}0.032 \\
(1.02)^{\mathrm{a}}\end{array}$ & $\begin{array}{c}0.031 \\
(1.02)^{\mathrm{a}}\end{array}$ \\
\hline ATM & $\begin{array}{c}0.009 \\
(1.01)^{\mathrm{a}}\end{array}$ & $\begin{array}{c}0.008 \\
(1.00)^{\mathrm{a}}\end{array}$ & $\begin{array}{c}0.008 \\
(1.00)^{\mathrm{a}}\end{array}$ & $\begin{array}{c}0.010 \\
(1.01)^{\mathrm{a}}\end{array}$ & $\begin{array}{c}0.022 \\
(1.01)^{\mathrm{a}}\end{array}$ & $\begin{array}{c}0.021 \\
(1.01)^{\mathrm{a}}\end{array}$ & $\begin{array}{c}0.035 \\
(1.02)^{\mathrm{a}}\end{array}$ & $\begin{array}{c}0.029 \\
(1.01)^{\mathrm{a}}\end{array}$ \\
\hline Bhakti & $\begin{array}{c}0.010 \\
(1.01)^{\mathrm{a}}\end{array}$ & $\begin{array}{c}0.009 \\
(1.00)^{\mathrm{a}}\end{array}$ & $\begin{array}{c}0.008 \\
(1.00)^{\mathrm{a}}\end{array}$ & $\begin{array}{c}0.010 \\
(1.01)^{\mathrm{a}}\end{array}$ & $\begin{array}{c}0.028 \\
(1.01)^{\mathrm{a}}\end{array}$ & $\begin{array}{c}0.035 \\
(1.02)^{\mathrm{a}}\end{array}$ & $\begin{array}{c}0.033 \\
(1.02)^{\mathrm{a}}\end{array}$ & $\begin{array}{c}0.029 \\
(1.01)^{\mathrm{a}}\end{array}$ \\
\hline Brahma & $\begin{array}{c}0.010 \\
(1.01)^{\mathrm{a}}\end{array}$ & $\begin{array}{c}0.008 \\
(1.00)^{\mathrm{a}}\end{array}$ & $\begin{array}{c}0.012 \\
(1.01)^{\mathrm{a}}\end{array}$ & $\begin{array}{c}0.011 \\
(1.01)^{\mathrm{a}}\end{array}$ & $\begin{array}{c}0.027 \\
(1.01)^{\mathrm{a}}\end{array}$ & $\begin{array}{c}0.030 \\
(1.02)^{\mathrm{a}}\end{array}$ & $\begin{array}{c}0.041 \\
(1.02)^{\mathrm{a}}\end{array}$ & $\begin{array}{c}0.028 \\
(1.01)^{\mathrm{a}}\end{array}$ \\
\hline Denim & $\begin{array}{c}0.010 \\
(1.01)^{\mathrm{a}}\end{array}$ & $\begin{array}{c}0.008 \\
(1.00)^{\mathrm{a}}\end{array}$ & $\begin{array}{c}0.009 \\
(1.00)^{\mathrm{a}}\end{array}$ & $\begin{array}{c}0.007 \\
(1.00)^{\mathrm{a}}\end{array}$ & $\begin{array}{c}0.033 \\
(1.02)^{\mathrm{a}}\end{array}$ & $\begin{array}{c}0.030 \\
(1.02)^{\mathrm{a}}\end{array}$ & $\begin{array}{c}0.041 \\
(1.02)^{\mathrm{a}}\end{array}$ & $\begin{array}{c}0.027 \\
(1.01)^{\mathrm{a}}\end{array}$ \\
\hline Rasi-665 & $\begin{array}{c}0.009 \\
(1.01)^{\mathrm{a}}\end{array}$ & $\begin{array}{c}0.005 \\
(1.00)^{\mathrm{a}}\end{array}$ & $\begin{array}{l}0.007 \\
\left(1.00^{\mathrm{a}}\right.\end{array}$ & $\begin{array}{c}0.007 \\
(1.00)^{\mathrm{a}}\end{array}$ & $\begin{array}{c}0.020 \\
(1.01)^{\mathrm{a}}\end{array}$ & $\begin{array}{c}0.021 \\
(1.01)^{\mathrm{a}}\end{array}$ & $\begin{array}{c}0.026 \\
(1.01)^{\mathrm{a}}\end{array}$ & $\begin{array}{c}0.024 \\
(1.01)^{\mathrm{a}}\end{array}$ \\
\hline Rasi-668 & $\begin{array}{c}0.009 \\
(1.00)^{\mathrm{a}}\end{array}$ & $\begin{array}{c}0.011 \\
(1.01)^{\mathrm{a}}\end{array}$ & $\begin{array}{c}0.011 \\
(1.01)^{\mathrm{a}}\end{array}$ & $\begin{array}{c}0.009 \\
(1.01)^{\mathrm{a}}\end{array}$ & $\begin{array}{c}0.034 \\
(1.02)^{\mathrm{a}}\end{array}$ & $\begin{array}{c}0.025 \\
(1.01)^{\mathrm{a}}\end{array}$ & $\begin{array}{c}0.029 \\
(1.01)^{\mathrm{a}}\end{array}$ & $\begin{array}{c}0.027 \\
(1.01)^{\mathrm{a}}\end{array}$ \\
\hline Sudarshan & $\begin{array}{c}0.009 \\
(1.01)^{\mathrm{a}}\end{array}$ & $\begin{array}{c}0.008 \\
(1.00)^{\mathrm{a}}\end{array}$ & $\begin{array}{c}0.009 \\
(1.00)^{\mathrm{a}}\end{array}$ & $\begin{array}{c}0.009 \\
(1.00)^{\mathrm{a}}\end{array}$ & $\begin{array}{c}0.035 \\
(1.02)^{\mathrm{a}}\end{array}$ & $\begin{array}{c}0.033 \\
(1.02)^{\mathrm{a}}\end{array}$ & $\begin{array}{c}0.038 \\
(1.02)^{\mathrm{a}}\end{array}$ & $\begin{array}{c}0.029 \\
(1.01)^{\mathrm{a}}\end{array}$ \\
\hline Yuva & $\begin{array}{c}0.012 \\
(1.01)^{\mathrm{a}}\end{array}$ & $\begin{array}{c}0.012 \\
(1.01)^{\mathrm{a}}\end{array}$ & $\begin{array}{c}0.012 \\
(1.01)^{\mathrm{a}}\end{array}$ & $\begin{array}{c}0.012 \\
(1.01)^{\mathrm{a}}\end{array}$ & $\begin{array}{c}0.043 \\
(1.02)^{\mathrm{a}}\end{array}$ & $\begin{array}{c}0.029 \\
(1.02)^{\mathrm{a}}\end{array}$ & $\begin{array}{c}0.035 \\
(1.02)^{\mathrm{a}}\end{array}$ & $\begin{array}{c}0.031 \\
(1.02)^{\mathrm{a}}\end{array}$ \\
\hline Control & $\begin{array}{c}0.010 \\
(1.01)^{\mathrm{a}}\end{array}$ & $\begin{array}{c}0.015 \\
(1.01)^{\mathrm{a}}\end{array}$ & $\begin{array}{c}0.016 \\
(1.01)^{\mathrm{a}}\end{array}$ & $\begin{array}{c}0.015 \\
(1.01)^{\mathrm{a}}\end{array}$ & $\begin{array}{c}0.046 \\
(1.02)^{\mathrm{a}}\end{array}$ & $\begin{array}{c}0.038 \\
(1.02)^{\mathrm{a}}\end{array}$ & $\begin{array}{c}0.033 \\
(1.02)^{\mathrm{a}}\end{array}$ & $\begin{array}{c}0.031 \\
(1.02)^{\mathrm{a}}\end{array}$ \\
\hline CD & 0.001 & 0.001 & 0.001 & 0.001 & 0.004 & 0.003 & 0.002 & 0.002 \\
\hline SE (m) & 0 & 0 & 0 & 0 & 0.001 & 0.001 & 0.001 & 0.001 \\
\hline
\end{tabular}

Figures in parentheses are square root transformed values; numbers followed by same superscript are not statistically different.

Table.3 Effect of test hybrid squares on weight of third and fourth instar larvae of S. litura. 


\begin{tabular}{|c|c|c|c|c|c|c|c|c|}
\hline \multirow{2}{*}{ Treatments } & \multicolumn{4}{|c|}{ Weight of third Instar larvae (g) } & \multicolumn{4}{|c|}{ Weight of fourth Instar larvae (g) } \\
\hline & 60 DAS & 75 DAS & 90 DAS & 125 DAS & 60 DAS & 75 DAS & 90 DAS & 125 DAS \\
\hline Ankur-3034 & $\begin{array}{c}0.076 \\
(1.04)^{\mathrm{a}}\end{array}$ & $\begin{array}{c}0.075 \\
(1.04)^{\mathrm{a}}\end{array}$ & $\begin{array}{c}0.096 \\
(1.05)^{\mathrm{a}}\end{array}$ & $\begin{array}{c}0.080 \\
(1.04)^{\mathrm{a}}\end{array}$ & $\begin{array}{c}0.198 \\
(1.10)^{\mathrm{a}}\end{array}$ & $\begin{array}{c}0.192 \\
(1.09)^{\mathrm{a}}\end{array}$ & $\begin{array}{c}0.196 \\
(1.09)^{\mathrm{a}}\end{array}$ & $\begin{array}{c}0.181 \\
(1.09)^{\mathrm{a}}\end{array}$ \\
\hline Ajeet-155 & $\begin{array}{c}0.099 \\
(1.05)^{\mathrm{a}}\end{array}$ & $\begin{array}{c}0.094 \\
(1.05)^{\mathrm{a}}\end{array}$ & $\begin{array}{c}0.107 \\
(1.05)^{\mathrm{a}}\end{array}$ & $\begin{array}{c}0.099 \\
(1.05)^{\mathrm{a}}\end{array}$ & $\begin{array}{c}0.222 \\
(1.11)^{\mathrm{a}}\end{array}$ & $\begin{array}{c}0.226 \\
(1.11)^{\mathrm{a}}\end{array}$ & $\begin{array}{c}0.234 \\
(1.11)^{\mathrm{a}}\end{array}$ & $\begin{array}{c}0.221 \\
(1.11)^{\mathrm{a}}\end{array}$ \\
\hline Chetak & $\begin{array}{c}0.088 \\
(1.04)^{\mathrm{a}}\end{array}$ & $\begin{array}{c}0.081 \\
(1.04)^{\mathrm{a}}\end{array}$ & $\begin{array}{c}0.082 \\
(1.04)^{\mathrm{a}}\end{array}$ & $\begin{array}{c}0.070 \\
(1.04)^{\mathrm{a}}\end{array}$ & $\begin{array}{c}0.216 \\
(1.10)^{\mathrm{a}}\end{array}$ & $\begin{array}{c}0.187 \\
(1.09)^{\mathrm{a}}\end{array}$ & $\begin{array}{c}0.215 \\
(1.10)^{\mathrm{a}}\end{array}$ & $\begin{array}{c}0.188 \\
(1.09)^{\mathrm{a}}\end{array}$ \\
\hline ATM & $\begin{array}{c}0.090 \\
(1.04)^{\mathrm{a}}\end{array}$ & $\begin{array}{c}0.069 \\
(1.03)^{\mathrm{a}}\end{array}$ & $\begin{array}{c}0.079 \\
(1.04)^{\mathrm{a}}\end{array}$ & $\begin{array}{c}0.065 \\
(1.03)^{\mathrm{a}}\end{array}$ & $\begin{array}{c}0.196 \\
(1.10)^{\mathrm{a}}\end{array}$ & $\begin{array}{c}0.199 \\
(1.10)^{\mathrm{a}}\end{array}$ & $\begin{array}{c}0.208 \\
(1.10)^{\mathrm{a}}\end{array}$ & $\begin{array}{c}0.193 \\
(1.09)^{\mathrm{a}}\end{array}$ \\
\hline Bhakti & $\begin{array}{c}0.091 \\
(1.05)^{\mathrm{a}}\end{array}$ & $\begin{array}{c}0.089 \\
(1.04)^{\mathrm{a}}\end{array}$ & $\begin{array}{c}0.064 \\
(1.03)^{\mathrm{a}}\end{array}$ & $\begin{array}{c}0.083 \\
(1.04)^{\mathrm{a}}\end{array}$ & $\begin{array}{c}0.235 \\
(1.11)^{\mathrm{a}}\end{array}$ & $\begin{array}{c}0.189 \\
(1.09)^{\mathrm{a}}\end{array}$ & $\begin{array}{c}0.214 \\
(1.10)^{\mathrm{a}}\end{array}$ & $\begin{array}{c}0.201 \\
(1.10)^{\mathrm{a}}\end{array}$ \\
\hline Brahma & $\begin{array}{c}0.086 \\
(1.04)^{\mathrm{a}}\end{array}$ & $\begin{array}{c}0.083 \\
(1.04)^{\mathrm{a}}\end{array}$ & $\begin{array}{c}0.083 \\
(1.04)^{\mathrm{a}}\end{array}$ & $\begin{array}{c}0.060 \\
(1.03)^{\mathrm{a}}\end{array}$ & $\begin{array}{c}0.224 \\
(1.11)^{\mathrm{a}}\end{array}$ & $\begin{array}{c}0.218 \\
(1.10)^{\mathrm{a}}\end{array}$ & $\begin{array}{c}0.207 \\
(1.10)^{\mathrm{a}}\end{array}$ & $\begin{array}{c}0.234 \\
(1.11)^{\mathrm{a}}\end{array}$ \\
\hline Denim & $\begin{array}{c}0.092 \\
(1.05)^{\mathrm{a}}\end{array}$ & $\begin{array}{c}0.085 \\
(1.04)^{\mathrm{a}}\end{array}$ & $\begin{array}{c}0.080 \\
(1.04)^{\mathrm{a}}\end{array}$ & $\begin{array}{c}0.055 \\
(1.03)^{\mathrm{a}}\end{array}$ & $\begin{array}{c}0.219 \\
(1.10)^{\mathrm{a}}\end{array}$ & $\begin{array}{c}0.215 \\
(1.10)^{\mathrm{a}}\end{array}$ & $\begin{array}{c}0.232 \\
(1.11)^{\mathrm{a}}\end{array}$ & $\begin{array}{c}0.227 \\
(1.10)^{\mathrm{a}}\end{array}$ \\
\hline Rasi-665 & $\begin{array}{c}0.074 \\
(1.04)^{\mathrm{a}}\end{array}$ & $\begin{array}{c}0.066 \\
(1.03)^{\mathrm{a}}\end{array}$ & $\begin{array}{c}0.077 \\
(1.04)^{\mathrm{a}}\end{array}$ & $\begin{array}{c}0.058 \\
(1.03)^{\mathrm{a}}\end{array}$ & $\begin{array}{c}0.199 \\
(1.10)^{\mathrm{a}}\end{array}$ & $\begin{array}{c}0.177 \\
(1.09)^{\mathrm{a}}\end{array}$ & $\begin{array}{c}0.191 \\
(1.09)^{\mathrm{a}}\end{array}$ & $\begin{array}{c}0.175 \\
(1.08)^{\mathrm{a}}\end{array}$ \\
\hline Rasi-668 & $\begin{array}{c}0.076 \\
(1.04)^{\mathrm{a}} \\
\end{array}$ & $\begin{array}{c}0.074 \\
(1.04)^{\mathrm{a}} \\
\end{array}$ & $\begin{array}{c}0.084 \\
(1.04)^{\mathrm{a}} \\
\end{array}$ & $\begin{array}{c}0.055 \\
(1.03)^{\mathrm{a}} \\
\end{array}$ & $\begin{array}{c}0.193 \\
(1.09)^{\mathrm{a}} \\
\end{array}$ & $\begin{array}{c}0.198 \\
(1.02)^{\mathrm{a}} \\
\end{array}$ & $\begin{array}{c}0.207 \\
(1.10)^{\mathrm{a}} \\
\end{array}$ & $\begin{array}{c}0.179 \\
(1.08)^{\mathrm{a}} \\
\end{array}$ \\
\hline Sudarshan & $\begin{array}{c}0.091 \\
(1.05)^{\mathrm{a}}\end{array}$ & $\begin{array}{c}0.088 \\
(1.04)^{\mathrm{a}}\end{array}$ & $\begin{array}{c}0.090 \\
(1.04)^{\mathrm{a}}\end{array}$ & $\begin{array}{c}0.082 \\
(1.04)^{\mathrm{a}}\end{array}$ & $\begin{array}{c}0.209 \\
(1.10)^{\mathrm{a}}\end{array}$ & $\begin{array}{c}0.197 \\
(1.09)^{\mathrm{a}}\end{array}$ & $\begin{array}{c}0.213 \\
(1.10)^{\mathrm{a}}\end{array}$ & $\begin{array}{c}0.177 \\
(1.09)^{\mathrm{a}}\end{array}$ \\
\hline Yuva & $\begin{array}{c}0.077 \\
(1.04)^{\mathrm{a}}\end{array}$ & $\begin{array}{c}0.076 \\
(1.04)^{\mathrm{a}}\end{array}$ & $\begin{array}{c}0.079 \\
(1.04)^{\mathrm{a}}\end{array}$ & $\begin{array}{c}0.084 \\
(1.04)^{\mathrm{a}}\end{array}$ & $\begin{array}{c}0.234 \\
(1.11)^{\mathrm{a}}\end{array}$ & $\begin{array}{c}0.194 \\
(1.09)^{\mathrm{a}}\end{array}$ & $\begin{array}{c}0.240 \\
(1.11)^{\mathrm{a}}\end{array}$ & $\begin{array}{c}0.223 \\
(1.11)^{\mathrm{a}}\end{array}$ \\
\hline control & $\begin{array}{c}0.095 \\
(1.05)^{\mathrm{a}} \\
\end{array}$ & $\begin{array}{c}0.089 \\
(1.04)^{\mathrm{a}} \\
\end{array}$ & $\begin{array}{c}0.084 \\
(1.04)^{\mathrm{a}} \\
\end{array}$ & $\begin{array}{c}0.083 \\
(1.04)^{\mathrm{a}}\end{array}$ & $\begin{array}{c}0.225 \\
(1.11)^{\mathrm{a}} \\
\end{array}$ & $\begin{array}{c}0.281 \\
(1.13)^{\mathrm{a}} \\
\end{array}$ & $\begin{array}{c}0.255 \\
(1.12)^{\mathrm{a}} \\
\end{array}$ & $\begin{array}{c}0.224 \\
(1.11)^{\mathrm{a}} \\
\end{array}$ \\
\hline CD & 0.005 & 0.004 & 0.003 & 0.005 & N.S. & 0.017 & 0.01 & 0.014 \\
\hline SE (m) & 0.002 & 0.002 & 0.001 & 0.002 & 0.006 & 0.006 & 0.003 & 0.005 \\
\hline
\end{tabular}

Figures in parentheses are square root transformed values; numbers followed by same superscript are not statistically different.

Table.4 Effect of test hybrid bolls on larval weight of S. litura. 


\begin{tabular}{|c|c|c|c|c|c|c|}
\hline \multirow{2}{*}{ Treatments } & \multicolumn{2}{|c|}{ Weight of second Instar larvae (g) } & \multicolumn{2}{|c|}{ Weight of third Instar larvae (g) } & \multicolumn{2}{|c|}{ Weight of fourth Instar larvae (g) } \\
\hline & 90 DAS & 125 DAS & 90 DAS & 125 DAS & 90 DAS & 125 DAS \\
\hline Ankur-3034 & $\begin{array}{c}0.035 \\
(1.02)^{\mathrm{a}}\end{array}$ & $\begin{array}{c}0.034 \\
(1.02)^{\mathrm{a}}\end{array}$ & $\begin{array}{c}0.090 \\
(1.04)^{\mathrm{a}}\end{array}$ & $\begin{array}{c}0.087 \\
(1.04)^{\mathrm{a}}\end{array}$ & $\begin{array}{c}0.234 \\
(1.11)^{\mathrm{a}}\end{array}$ & $\begin{array}{c}0.231 \\
(1.11)^{\mathrm{a}}\end{array}$ \\
\hline Ajeet-155 & $\begin{array}{c}0.045 \\
(1.02)^{\mathrm{a}}\end{array}$ & $\begin{array}{c}0.037 \\
(1.02)^{\mathrm{a}}\end{array}$ & $\begin{array}{c}0.081 \\
(1.04)^{\mathrm{a}}\end{array}$ & $\begin{array}{c}0.092 \\
(1.05)^{\mathrm{a}}\end{array}$ & $\begin{array}{c}0.267 \\
(1.13)^{\mathrm{a}}\end{array}$ & $\begin{array}{c}0.335 \\
(1.16)^{\mathrm{a}}\end{array}$ \\
\hline Chetak & $\begin{array}{c}0.029 \\
(1.01)^{\mathrm{a}}\end{array}$ & $\begin{array}{c}0.028 \\
(1.01)^{\mathrm{a}}\end{array}$ & $\begin{array}{c}0.087 \\
(1.04)^{\mathrm{a}}\end{array}$ & $\begin{array}{c}0.083 \\
(1.04)^{\mathrm{a}}\end{array}$ & $\begin{array}{c}0.211 \\
(1.10)^{\mathrm{a}}\end{array}$ & $\begin{array}{c}0.228 \\
(1.11)^{\mathrm{a}}\end{array}$ \\
\hline ATM & $\begin{array}{c}0.033 \\
(1.02)^{\mathrm{a}}\end{array}$ & $\begin{array}{c}0.029 \\
(1.01)^{\mathrm{a}}\end{array}$ & $\begin{array}{c}0.084 \\
(1.04)^{\mathrm{a}}\end{array}$ & $\begin{array}{c}0.089 \\
(1.04)^{\mathrm{a}}\end{array}$ & $\begin{array}{c}0.204 \\
(1.10)^{\mathrm{a}}\end{array}$ & $\begin{array}{c}0.267 \\
(1.13)^{\mathrm{a}}\end{array}$ \\
\hline Bhakti & $\begin{array}{c}0.028 \\
(1.01)^{\mathrm{a}}\end{array}$ & $\begin{array}{c}0.028 \\
(1.01)^{\mathrm{a}}\end{array}$ & $\begin{array}{c}0.086 \\
(1.04)^{\mathrm{a}}\end{array}$ & $\begin{array}{c}0.086 \\
(1.04)^{\mathrm{a}}\end{array}$ & $\begin{array}{c}0.255 \\
(1.12)^{\mathrm{a}}\end{array}$ & $\begin{array}{c}0.271 \\
(1.13)^{\mathrm{a}}\end{array}$ \\
\hline Brahma & $\begin{array}{c}0.030 \\
(1.02)^{\mathrm{a}}\end{array}$ & $\begin{array}{c}0.022 \\
(1.01)^{\mathrm{a}}\end{array}$ & $\begin{array}{c}0.077 \\
(1.04)^{\mathrm{a}}\end{array}$ & $\begin{array}{c}0.062 \\
(1.03)^{\mathrm{a}}\end{array}$ & $\begin{array}{c}0.243 \\
(1.12)^{\mathrm{a}}\end{array}$ & $\begin{array}{c}0.218 \\
(1.10)^{\mathrm{a}}\end{array}$ \\
\hline Denim & $\begin{array}{c}0.029 \\
(1.01)^{\mathrm{a}}\end{array}$ & $\begin{array}{c}0.031 \\
(1.02)^{\mathrm{a}}\end{array}$ & $\begin{array}{c}0.084 \\
(1.04)^{\mathrm{a}}\end{array}$ & $\begin{array}{c}0.063 \\
(1.03)^{\mathrm{a}}\end{array}$ & $\begin{array}{c}0.234 \\
(1.11)^{\mathrm{a}}\end{array}$ & $\begin{array}{c}0.253 \\
(1.12)^{\mathrm{a}}\end{array}$ \\
\hline Rasi-665 & $\begin{array}{c}0.024 \\
(1.01)^{\mathrm{a}}\end{array}$ & $\begin{array}{c}0.017 \\
(1.01)^{\mathrm{a}}\end{array}$ & $\begin{array}{c}0.073 \\
(1.04)^{\mathrm{a}}\end{array}$ & $\begin{array}{c}0.053 \\
(1.03)^{\mathrm{a}}\end{array}$ & $\begin{array}{c}0.191 \\
(1.09)^{\mathrm{a}}\end{array}$ & $\begin{array}{c}0.249 \\
(1.12)^{\mathrm{a}}\end{array}$ \\
\hline Rasi-668 & $\begin{array}{c}0.028 \\
(1.01)^{\mathrm{a}}\end{array}$ & $\begin{array}{c}0.021 \\
(1.01)^{\mathrm{a}}\end{array}$ & $\begin{array}{c}0.086 \\
(1.04)^{\mathrm{a}}\end{array}$ & $\begin{array}{c}0.062 \\
(1.03)^{\mathrm{a}}\end{array}$ & $\begin{array}{c}0.217 \\
(1.10)^{\mathrm{a}}\end{array}$ & $\begin{array}{c}0.224 \\
(1.11)^{\mathrm{a}}\end{array}$ \\
\hline Sudarshan & $\begin{array}{c}0.033 \\
(1.02)^{\mathrm{a}}\end{array}$ & $\begin{array}{c}0.043 \\
(1.02)^{\mathrm{a}}\end{array}$ & $\begin{array}{c}0.083 \\
(1.04)^{\mathrm{a}}\end{array}$ & $\begin{array}{c}0.082 \\
(1.04)^{\mathrm{a}}\end{array}$ & $\begin{array}{c}0.191 \\
(1.09)^{\mathrm{a}}\end{array}$ & $\begin{array}{c}0.313 \\
(1.15)^{\mathrm{a}}\end{array}$ \\
\hline Yuva & $\begin{array}{c}0.039 \\
(1.02)^{\mathrm{a}}\end{array}$ & $\begin{array}{c}0.042 \\
(1.02)^{\mathrm{a}}\end{array}$ & $\begin{array}{c}0.076 \\
(1.04)^{\mathrm{a}}\end{array}$ & $\begin{array}{c}0.086 \\
(1.04)^{\mathrm{a}}\end{array}$ & $\begin{array}{c}0.219 \\
(1.104)^{\mathrm{a}}\end{array}$ & $\begin{array}{c}0.221 \\
(1.11)^{\mathrm{a}}\end{array}$ \\
\hline Control & $\begin{array}{c}0.030 \\
(1.02)^{\mathrm{a}}\end{array}$ & $\begin{array}{c}0.031 \\
(1.02)^{\mathrm{a}}\end{array}$ & $\begin{array}{c}0.090 \\
(1.04)^{\mathrm{a}}\end{array}$ & $\begin{array}{c}0.087 \\
(1.04)^{\mathrm{a}}\end{array}$ & $\begin{array}{c}0.252 \\
(1.12)^{\mathrm{a}}\end{array}$ & $\begin{array}{c}0.371 \\
(1.17)^{\mathrm{a}}\end{array}$ \\
\hline CD & 0.004 & 0.003 & 0.005 & 0.004 & 0.006 & 0.008 \\
\hline SE (m) & 0.001 & 0.001 & 0.002 & 0.001 & 0.002 & 0.003 \\
\hline
\end{tabular}

Figures in parentheses are square root transformed values; numbers followed by same superscript are not statistically different. 
However, statistically all eleven BG-II cotton hybrids were on par with each other. Rasi-665 BG-II cotton recorded minimum weight of first, second, third and fourth instar in between 60 and 125 DAS. The present results suggest that dual toxin Bt cottons will provide substantially better control of S. litura.

\section{References}

Adamczyk, J.J., Hardee D, Adams L, Sumerford DV, Correlating differences In larval survival and development of bollworms (Lepidoptera: Noctuidae) and fall armyworms (Lepidoptera : Noctuidae) to differential expression of Cry 1 Ac(c) endotoxin in various plant parts among commercial cultivars of transgenic Bacillus thuringiensis cotton, Journal of Economic Entomology, 94 (2001) 284.

Gomez, K.A., Gomez AA, Statistical procedures for Agricultural Research, Second edition. John wiley and Sons, New York, (1984) 582.

Gore, J., Leonard BR, Adamczyk JJ, Bollworm (Lepidoptera: Noctuidae) survival on Bollgard and Bollgard-II cotton flower bud and flower components, Journal of Economic Entomology, 94 (2001) 1445.

Jackson, R.E., Bradley JRJr, Burd AD, Van DJW, Field and greenhouse performance of bollworm on Bollgard II cotton genotypes, Proceedings of Beltwide Cotton Conference. San Antonio, Jan., 9-13. (2004) 1048.

Jia, S.R., Guo SD, An BC, Transgenic Cotton. Science Publishing house of China, Beijing, (2001).

Naik, V.C.B., Prasad NVVSD, Rao GR, Effect of $\mathrm{Bt}$ cotton on survival and development of tobacco caterpillar, Spodoptera litura (fabricius) on different events of Bt hybrids, African Journal of Agricultural Research, 8 (2013) 5538.

Prasad, J.V., Sreedhar U, Life parameters of tobacco caterpillar, Spodoptera litura as influenced by transgenic $(\mathrm{Bt})$ cotton hybrids, Indian Journal of Entomology, 73(2011) 312.

Soujanya, P.L., Prasad NVVSD, Rao PA, Gopinath M, Larval survival and development of Spodoptera litura (f.) on dual toxin Bt cotton (Cry $1 \mathrm{Ac}+2$ $\mathrm{Ab})$ and single toxin Bt cotton (Cry 1 Ac), Indian Journal of Entomology, 73 (2011) 19.

\section{How to cite this article:}

Ramanjali, T., T.V.K. Singh and Narendra Reddy, C. 2017. Effect of BG-II Cotton Hybrids and Non Bt Cotton on Weight of Different Instars of Spodoptera litura (FAB.). Int.J.Curr.Microbiol.App.Sci. 6(10): 2170-2177. doi: https://doi.org/10.20546/ijcmas.2017.610.257 